\title{
Terahertz pulse generation by multi-color laser fields with linear vs. circular polarization
}

\author{
A. Stathopulos ${ }^{1,2}$, C. Tailliez ${ }^{1,2}$, D. Buožius ${ }^{4}$, I. Babushkin ${ }^{5,6}$, V. Vaičaitis ${ }^{4}$, S. Skupin $^{3}$, and L. Bergé ${ }^{1,2}$ \\ 1. CEA-DAM, DIF, 91297 Arpajon, France \\ 2. Université Paris-Saclay, CEA, LMCE, 91680 Bruyères-le-Châtel, France \\ 3. Institut Lumière Matière, UMR 5306 Université Lyon 1 - CNRS, Université de Lyon, 69622 Villeurbanne, France \\ 4. Institute of Quantum Optics, Leibniz University Hannover, Welfengarten 1 30167, Hannover, Germany \\ 5. Cluster of Excellence PhoenixD (Photonics, Optics, and Engineering-Innovation Across Disciplines), 30167 Hannover, Germany
}

Terahertz (THz) radiation produced by ultrafast laser sources has recently become a hot topic with many innovative applications in molecular spectroscopy, medical imaging or homeland security [1]. Efficient THz emitters can be obtained by focusing into air a 2-colors femtosecond light pulse, composed of fundamental $(\mathrm{FH})$ and second (SH) harmonics, in order to create a plasma spot that acts as a frequency converter [2].

On the one hand, recent studies [3,4] showed that increasing the number of laser harmonics (called colors) until shaping the laser temporal profile with a sawtooth waveform can lead to noticeable enhancement in the laser-to-THz conversion efficiency driven by photocurrents. On the other hand, changing the polarization state of the FH and SH components can also improve the THz performances of laser-plasma emitters. In particular, it was experimentally evidenced [5] that circularly-polarized 2-colors pulses with same helicity (CP-S) can deliver $\sim 3-5$ times higher THz powers than their linearly-polarized, parallel pulses (LP-P). In [6], theoretical evaluations showed that the direct dependency of the drift velocity acquired by photo-ionized electrons on the FH pump amplitude and longer ionization sequences explain this increase in THz power, which is refound below in Figs. 1(a,b) from a local current model and in Fig. 1(e) (dashed curves) from comprehensive 3D computations.

Here we combine the advantages of adding more colors (up to 10) arranged within a sawtooth wave shape and circular polarization to achieve much higher THz energy yields. In air, whose ionization of $\mathrm{O}_{2}$ molecules saturates early along the pulse history, we report gain factors $>4$ by using 10-colors LP-P sawtooth field and $>2$ reached by 10-colors CP-S sawtooth pulses compared with their 2-colors counterparts [see Figs. 1(c,d) and 1(e)]. Surprisingly, the LP-P fields become more efficient than CP-S ones by increasing the number of colors above 6 , which we attribute to larger electron drift velocities acquired at the ionization instants.
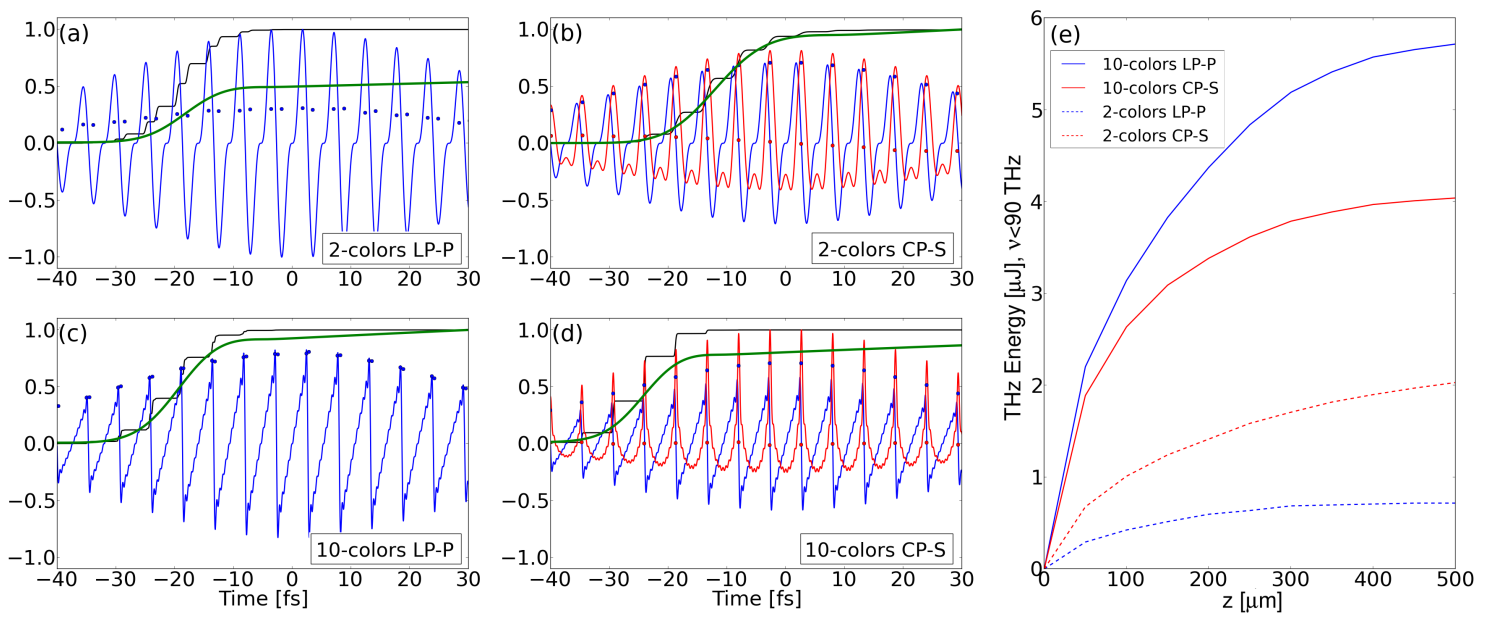

Figure 1 Electron density $N_{e} / N_{a}$ (black curves), laser fields $E_{L} / E_{\max }$ (blue, red curves), electron drift velocity at ionization times (blue, red dots) and THz energy (green curves) versus time for 2-colors (a) LP-P; (b) CP-S pulses, and 10-colors (c) LP-P and (d) CP-S pulses ionizing air with $200 \mathrm{TW} / \mathrm{cm}^{2}$ intensity, $1600 \mathrm{~nm} \mathrm{FH}$ and $50 \mathrm{fs}$ FWHM (local current model). Quantites are normalized to their maximum at constant number of colors $\left[E_{\max }=45\right.$ and $64 \mathrm{GV} / \mathrm{m}$ in $(\mathrm{a}, \mathrm{b})$ and $(\mathrm{c}, \mathrm{d})$, respectively; $N_{a}=5.4 \times 10^{18} \mathrm{~cm}^{-3}$ ]. (e) shows the THz energy yield obtained from 3D unidirectional computations for the same pulses.

These performances being sensitive to the ionization dynamics, we shall address pulse configurations leading to different ionization yields, including those of nitrogen, argon, helium, and their multiply ionized states.

\section{References}

[1] M. Tonouchi, "Cutting-edge terahertz technology," Nat. Photon. 1, 97 (2007).

[2] K. Y. Kim et al., "Coherent control of terahertz supercontinuum generation in ultrafast laser-gas interactions," Nat. Photon. 2, 605 (2008).

[3] P. Gonzalez de Alaiza Martinez et al., "Boosting Terahertz Generation in Laser-Field Ionized Gases Using a Sawtooth Wave Shape," Phys. Rev. Lett. 114, 183901 (2016).

[4] V. Vaicaitis et al., "Terahertz radiation generation by three-color laser pulses in air filament," J. Appl. Phys. 125, 173103 (2019).

[5] C. Meng et al., "Enhancement of THz radiation by using circularly polarized two-color laser fields," Appl. Phys. Lett. 109, 131105 (2016).

[6] C. Tailliez et al., "Terahertz pulse generation by two-color laser fields with circular polarization," New J. Phys. 22, 103038 (2020). 